\title{
Influencia de los cambios de presión atmosférica y otras variables meteorológicas en la incidencia de la hemorragia subaracnoidea
}

\author{
E. Baño-Ruiz; J. Abarca-Olivas; J.M. Duart-Clemente; F. Ballenilla-Marco*; P. García** y C. Botella-Asunción
}

Servicios de Neurocirugía, Neurorradiología* y Medicina Preventiva**. Hospital General Universitario de Alicante.

Resumen

La hemorragia subaracnoidea espontánea es una patología que habitualmente se relaciona con factores de riesgo, tales como la hipertensión arterial y/o el tabaquismo. Sin embargo, existen otras variables, como los cambios meteorológicos, que aunque la experiencia apunta como posibles responsables de un aumento de la incidencia, todavía no están establecidas como tales.

Objetivo. Investigar la influencia de los cambios meteorológicos, ya sean variaciones estacionales o cambios de presión atmosférica, en la incidencia de la hemorragia subaracnoidea.

Método. Se consideran todos los casos de hemorragia subaracnoidea espontánea desde el 1 de enero de 1997 al 31 de marzo de 2008, realizando un registro de aspectos demográficos (edad, sexo) y antecedentes médicos (hipertensión arterial, diabetes mellitus, patología cerebrovascular, tabaquismo), así como de las cifras de presión atmosférica (en hectopascales, hPa), temperatura y humedad relativa diarias, a lo largo de dicho periodo, con un cálculo de los valores máximo, medio y mínimo, así como de las diferencias de presión entre días consecutivos.

Resultados. El análisis de los 173 casos recogidos mostró un predominio de la hemorragia subaracnoidea en el sexo femenino, especialmente la de origen aneurismático, siendo la hipertensión arterial, el principal factor de riesgo relacionado. No se demostraron diferencias significativas entre los días con y sin hemorragia, en relación a la variación de presión atmosférica (p 0.463), temperatura ( $p 0.381$ ), humedad relativa ( $p$ 0.595) a lo largo del día.

Conclusión. En nuestro medio las variaciones de presión atmosférica no se relacionan con un aumento de la incidencia de la hemorragia subaracnoidea, produciéndose ésta con cambios de apenas 1,7 (1-3) hPa en el día, sin que se observe una clara tendencia estacional.

Recibido: 28-12-08. Aceptado: 17-05-09
PALABRAS CLAVE: Hemorragia subaracnoidea. Presión atmosférica. Temperatura. Humedad.

Summary

Spontaneous subarachnoid hemorrhage is a pathology usually related to risk factors, such as arterial hypertension and/or nicotine poisoning. Nevertheless, other variables exist, like meteorological changes, that although the experience aims as possible responsible for an increase of the incidence, are still not established like such.

Objetive. It is to investigate the influence of the meteorological changes, either seasonal variations or changes of atmospheric pressure, in the incidence of the subarachnoid hemorrhage.

Method. All the cases of spontaneous subarachnoid hemorrhage are considered from the 1th of January from 1997 to the 31th of March of 2008, making a medical registry of demographic aspects (age, sex) and antecedents (arterial hypertension, diabetes mellitus, cerebral ischemia, nicotine poisoning), as well as numbers of atmospheric pressure (in hectopascales, $\mathrm{hPa}$ ), daily temperature and relative humidity, throughout this period, with a calculation of the maximum, average and minimum values, as well as pressure differentials between consecutive days.

Results. The analysis of the 173 gathered cases showed a predominance of the subarachnoid hemorrhage in female, specially the aneurismal ones, being arterial hypertension, the main factor of related risk. Significant differences between the days with and without hemorrhage were not demonstrated, in relation to the variation of atmospheric pressure $(p 0,463)$, pressure temperature $(p 0,381)$ and relative humidity $(p$ $0,595)$ throughout the day.

Conclusion. In our element, the variations of atmospheric pressure are not related to an increase of incidence of the subarachnoid hemorrhage. Instead of this, cases of subarachnoid hemorrhage take place with changes of hardly $1.7(1-3) \mathrm{hPa}$ in the day, without 
observing a clear seasonal tendency.

KEY WORDS: Subarachnoid. Atmospheric. Pressure. Temperature. Humidity.

\section{Introducción}

La mayor parte de los casos de hemorragia subaracnoidea son secundarios a traumatismos craneoencefálicos. Sin embargo, en un número considerable de casos, que en algunas series se cifra en torno a 6-8 casos por 100.000 habitantes/año ${ }^{7}$, sucede de forma espontánea, con una mortalidad próxima a un $45 \%{ }^{17}$.

Existen factores de riesgo conocidos y aceptados como la hipertensión arterial y el tabaquismo ${ }^{5}$, entre otros (malformaciones arteriovenosas, tumores, disección arterias cerebrales, rotura de arterias superficiales, etc.) y otras variables que, a pesar de no estar tan establecidas, se consideran como posibles responsables de las variaciones en la incidencia de esta patología.

Diversos estudios abordan la influencia de las variables meteorológicas, con resultados muy contradictorios al respecto, encontrando que las variaciones en la incidencia de la HSA se relacionan con cambios de presión atmosférica entre los días hemorrágicos y no hemorrágicos en algunos de los estudios ${ }^{1,3,6,9,22}$, mientras que en otros la distribución de los casos no depende de la misma e incluso no sigue un patrón estacional ${ }^{2,19,20}$.

El objetivo de este estudio fue describir el patrón de presentación de HSA en el Servicio de Neurocirugía del Hospital General Universitario de Alicante así como determinar si éste se relaciona con los cambios meteorológicos.

\section{Material y métodos}

Se ha realizado un estudio retrospectivo de los casos de hemorragia subaracnoidea (HSA) espontánea, confirmada por técnicas de neuroimagen, principalmente tomografía computarizada, ingresados en el Servicio de Neurocirugía del Hospital General Universitario de Alicante durante el período comprendido entre enero de 1997 y marzo de 2008.

Los casos presentados fueron incluidos independientemente del grado clínico o radiológico, excluyendo únicamente a aquéllos debidos a traumatismo craneoencefálico. A todos los pacientes considerados en el estudio se les realizó una angiografía, siendo clasificados en HSA aneurismática (cuando se hallaba la presencia de uno o más aneurismas) o no aneurismática, en los casos restantes, sin tener en cuenta el manejo terapéutico posterior.

Se registraron variables demográficas (edad, sexo) y antecedentes médicos (hipertensión arterial, diabetes mellitus, tabaquismo y antecedentes de patología vascular, entre los que se consideran: HSA previa, aneurisma ya conocido e ictus isquémico o hemorrágico previos).

Asimismo, se consideró la fecha de ingreso como el día en que se produjo la hemorragia, teniendo en cuenta además, la estación del año en que se encontraba (Invierno [21 diciembre - 19 marzo], Primavera [20 marzo - 20 junio], Verano [21 junio - 22 septiembre] y Otoño [23 septiembre - 20 diciembre]). El dato del día de sangrado inicial se obtuvo de la hoja de entrada en el Servicio de Urgencias donde, en todos los casos, quedaba explícitamente reflejado por el médico de urgencias.

Los datos referentes a las variables atmosféricas fueron cedidos por el Centro Meteorológico Territorial de la Comunidad Valenciana, tomados en la Estación de Ciudad Jardín en Alicante.

Los valores de presión atmosférica se presentan expresados en hectopascales (hPa) y son resultado de mediciones realizadas de forma diaria a las 00:00 horas, 7:00 horas, 13: 00 horas y 18:00 horas, a partir de los cuales se determinaron los valores máximos, mínimos y medios (media aritmética de los cuatro valores de presión atmosférica tomados cada día), que permitieron el cálculo de la variación de la presión atmosférica en el día de la hemorragia (resultante de restar la mínima presión registrada en el día a la máxima presión en el mismo), así como la variación de la presión atmosférica máxima, mínima y media entre el día de la hemorragia y el día previo.

Los valores de temperatura se expresan en grados centígrados $\left({ }^{\circ} \mathrm{C}\right)$ y fueron registrados a las 00:00, 7:00, 13:00 y 18:00 horas de forma diaria, con registro de los valores máximo, mínimo y medio (media aritmética de los cuatro valores de temperatura tomados cada día) y cálculo de la variación de la temperatura en el día de la hemorragia (resultante de restar la mínima temperatura registrada en el día a la máxima temperatura) y las variaciones de la temperatura máxima, mínima y media entre el día de la hemorragia y el precedente.

De forma similar se procedió con los valores de humedad relativa, expresados en porcentaje (\%) y registrados en los mismos intervalos horarios anteriormente citados, considerando las cifras máxima, media y mínima, y deduciendo la variación de la humedad relativa en el día de la hemorragia (sustrayendo la mínima humedad relativa a la máxima registrada en dicho día) e igualmente las variaciones de la humedad relativa máxima, mínima y media entre el día de la hemorragia y el día previo.

El análisis de los datos se realizó con el programa informático SPSS versión 11.0. Se realizó un análisis descriptivo de las características de los pacientes incluidos. Para describir las variables cualitativas se utilizó la frecuencia absoluta y la relativa de cada uno de los valores de las variables; y para describir las variables cuantitativas se utilizó la media y la desviación estándar (distribución 
Tabla I

Distribución de casos de hemorragia subaracnoidea según variables demográficas

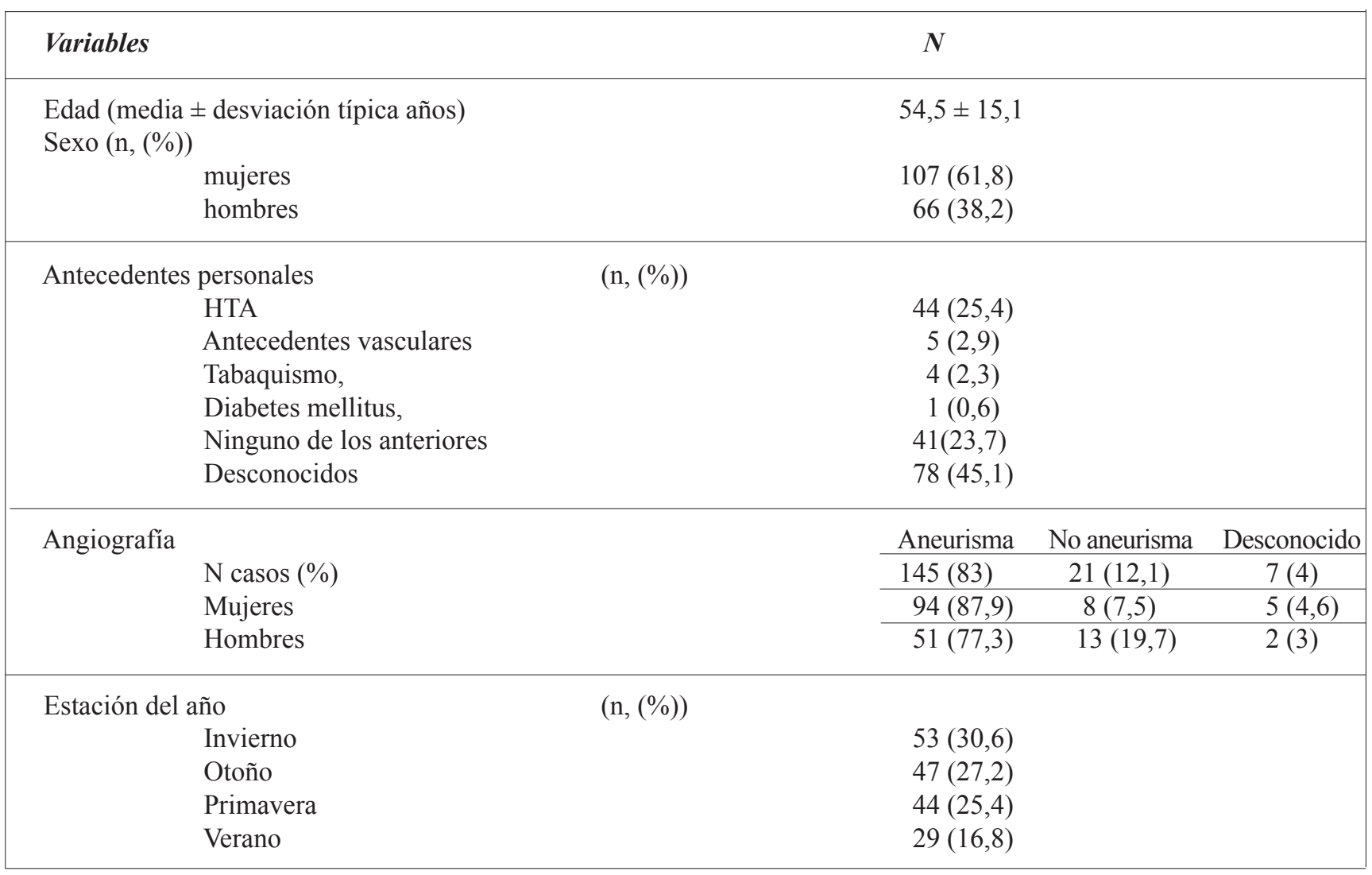

n: número de casos, HTA: hipertensión arterial

paramétrica) y la mediana y los percentiles 25 y 75 (distribución no paramétrica).

Para determinar la relación de las variaciones en la incidencia de casos de HSA con las variables cualitativas se emplearon las pruebas de Chi-cuadrado, mientras que para el análisis de las restantes variables cuantitativas, se emplearon pruebas no paramétricas como U de MannWhitney. En todos los contrastes de hipótesis referidos anteriormente se utilizara un nivel de significación estadística de $\mathrm{p}<0.05$.

\section{Resultados}

A lo largo del período de estudio, un total de 4.108 días, se registraron 173 casos de HSA espontánea, con una edad media (expresada como media \pm desviación típica) de 54,5 $\pm 15,1$ años y predominio del sexo femenino con 107 mujeres $(61,8 \%)$ frente a 66 hombres $(38,2 \%)$. Respecto a los antecedentes personales, de los 173 pacientes, $44(25,4 \%)$ presentaban HTA, antecedente médico más frecuente, 5 $(2,9 \%)$ antecedentes vasculares, $4(2,3 \%)$ tabaquismo, 1
$(0,6 \%)$ diabetes mellitus, con $41(23,7 \%)$ que no presentaban ninguno de los mencionados anteriormente, además de 78 pacientes $(45,1 \%)$ que no fueron incluidos por desconocimiento de sus antecedentes.

La realización de angiografías permitió la clasificación en 145 casos $(83,8 \%)$ de HSA aneurismática frente a 21 casos $(12,1 \%)$ de HSA no aneurismática y 7 casos $(4 \%)$ no considerados por desconocer el resultado de la prueba. En relación a la frecuencia acumulada por sexos, nuevamente se observa un predominio femenino de hemorragia aneurismática encontrando que, de los 107 casos descritos en este grupo, $87,9 \%$ de ellos son aneurismáticos, 7,5\% angiográficamente negativos y $4,6 \%$ desconocidos. De los 66 casos en el grupo masculino, 77,3\% presentaban aneurisma, $19,7 \%$ resultaron negativos y $3 \%$ desconocidos.

El análisis de la distribución por estaciones del año, no reflejó un claro predomino, pues del total de casos, el $30,6 \%$ se distribuyen en invierno, seguidos en frecuencia, por un $27,2 \%$ en otoño, $25,4 \%$ en primavera y $16,8 \%$ en verano. (Tabla I).

En relación a las variables meteorológicas (tabla II), 
Tabla II

Distribución de casos de hemorragia subaracnoidea según variables meteorológicas

\begin{tabular}{|cc|}
\hline Variables meteorológicas (variaciones) & mediana $\boldsymbol{y}\left(\boldsymbol{P}_{25}-\boldsymbol{P}_{50}\right)$ \\
\hline Presión atmosférica $\left[\right.$ mediana y $\left.\left(\mathrm{P}_{25}, \mathrm{P}_{50}\right) \mathrm{hPa}\right]$ & $1,7(1-3)$ \\
Diaria & $0,6(-2-3)$ \\
Máxima & $0,1(-2-3)$ \\
Mínima & $0,3(-2-3)$ \\
Media & \\
\hline Temperatura $\left({ }^{\circ} \mathrm{C}\right)$ & $10,2(-2-3)$ \\
Diaria & $0,00(-1--1)$ \\
Máxima & $-0,2(-1-1)$ \\
Mínima & $0,2(-3-4)$ \\
Media & \\
\hline Humedad relativa (\%) & $25,0(20-34)$ \\
Diaria & $-1,0(-7.5-7)$ \\
Máxima & $-1,0(-9-8)$ \\
Mínima & $-1,0(-10-9)$ \\
\hline
\end{tabular}

hPa: hectopascales; ${ }^{\circ} \mathrm{C}$ : grados centígrados

Tabla III

Comparación de la distribución por estaciones de los días con y sin casos de hemorragia subaracnoidea

\begin{tabular}{|c|c|c|c|c|}
\hline \multicolumn{5}{|c|}{ Días hemorrágicos } \\
\hline Estación & & $\begin{array}{c}\text { Sí } \\
(\mathrm{n}, \%)\end{array}$ & $\begin{array}{c}\text { No } \\
(n, \%)\end{array}$ & $P$ \\
\hline & Invierno & $49(4,6)$ & $1011(95,4)$ & \\
\hline & Otoño & $42(4,1)$ & $993(95,9)$ & 0,2 \\
\hline & Primavera & $30(2,9)$ & $1004(97,1)$ & \\
\hline & Verano & $43(4,0)$ & $936(95,6)$ & \\
\hline
\end{tabular}

n: número de casos

la presión atmosférica mostró variaciones medidas en términos de mediana $\left(\mathrm{P}_{25}, \mathrm{P}_{50}\right)$ diarias de $1,7(1,3) \mathrm{hPa}$, así como variaciones de las presiones máximas de $0,6(-2,3)$, mínimas de $0,1(-2,3)$ y medias de $0,3(-2,3)$ entre días correlativos.

La temperatura registró variaciones diarias de 10,2 (-2, $3)^{\circ} \mathrm{C}$, con diferencias entre días consecutivos de las temperaturas máxima, mínima y media de, $0,00(-1,-1),-0,2(-1$, 1) y $0,2(-3,4)$ respectivamente.

De forma similar, la humedad relativa presentó variaciones diarias de hasta $25,0 \%(20,34)$, con variaciones en los valores máximos de $-1,00(-7.5,7)$, mínimos de $-1,00 \%$ $(-9,8)$ y medios de $-1,0 \%(-10,9)$.

Para determinar si estas variaciones eran factores de riesgo para un aumento de la incidencia de HSA, se establecieron las diferencias en las tendencias de cambio de las variables meteorológicas entre los días en los que se producían casos de HSA y los días no hemorrágicos.

Teniendo en cuenta la distribución por estaciones tanto en el grupo de los días no hemorrágicos (tabla III) (Invierno 1011 días (95,4\%), Primavera 993 días $(95,9 \%)$, Verano 1004 días $(97,1 \%)$ y Otoño 936 días $(95,6 \%)$ ), como en el grupo de los días hemorrágicos (Invierno 49 días (4,6\%), Primavera 42 días $(4,1 \%)$, Verano 30 días $(2,9 \%)$ y Otoño 43 días $(4,0 \%))$, los días se repartían de forma equitativa entre las diversas estaciones, encontrando una tendencia descendente en el número de casos registrados en verano, que no alcanza la significación estadística (p: 0,193). 
Tabla IV

Cambios registrados de las variables meteorológicas entre días correlativos, en los días con y sin casos de hemorragia subaracnoidea

\begin{tabular}{|cccc|}
\hline Variables (variaciones) & Sí & Días hemorrágicos & \\
& $\left(\mathrm{P}_{25}, \mathrm{P}_{50}\right)$ & $\mathrm{No}$ & \\
\hline Presión (hPa) & & & \\
Diaria & $1,7(1-3)$ & $1,6(1-3)$ & N.S. \\
Máxima & $0,4(-2-3)$ & $0,1(-2-2)$ & N.S. \\
Mínima & $0,1(-2-3)$ & $0,1(-2-2)$ & N.S \\
Media & $-0,2(-2-3)$ & $-0,1(-2-2)$ & N.S. \\
\hline Temperatura $\left({ }^{\circ}\right.$ C) & & & N.S. \\
Diaria & $10(8-12)$ & $10(8-12)$ & 0,07 \\
Máxima & $0,0(-1-1)$ & $0,0(-1-1)$ & N.S. \\
Mínima & $-0,2(-1-1)$ & $0,0(-1-1)$ & $<0,05$ \\
Media & $0,3(-3-4)$ & $-0,3(-3-3)$ & N.S \\
\hline Humedad relativa(\%) & & & N.S \\
Diaria & $25(20-33)$ & $26(19-34)$ & N.S \\
Máxima & $-1,0(-8-7)$ & $1,0(-6-7)$ & N.S \\
Mínima & $0,0(-8-8)$ & $0,0(-8-9)$ & \\
Media & $-1,0(-11-9)$ & $0,0(-10-10)$ & \\
\hline
\end{tabular}

N.S.: estadísticametne no significativo; $\mathrm{hPa}$ : hectopascales; ${ }^{\circ} \mathrm{C}$ : grados centígrados

Al comparar las variaciones de presión atmosférica entre días correlativos en el grupo de los días HSA respecto al de los no hemorrágicos (tabla IV) apenas se han encontrado diferencias, que además no han resultado estadísticamente significativas tanto para las variaciones de presión diaria $(1,7(1-3)$ vs $1,6(1-3) \mathrm{hPa}, \mathrm{p} 0,463)$, presión máxima $0,4((-2)-3)$ vs $0,1((-2)-2) \mathrm{hPa}, \mathrm{p} 0,285)$, presión mínima $(0,1((-2)-3)$ vs $0,1((-2)-2) \mathrm{hPa}, \mathrm{p} 0,381)$ así como de presión media $-0,2((-2)-3)$ vs $-0,1((-2)-2)$ hPa, p 0,334).

Por otro lado, el análisis comparativo de las temperaturas tomadas en los días hemorrágicos frente a los no hemorrágicos reveló una situación similar a la de la presión atmosférica, sin alcanzar la significación estadística igualmente, para las variaciones de la temperatura diariamente [10 (8 - 12) vs $\left.10(8-12){ }^{\circ} \mathrm{C}, \mathrm{p} 0,381\right]$, máxima [0,0 (-1 - 1) vs $0,0((-1)-1)^{\circ} \mathrm{C}$ p 0,074$]$ y mínima $[-0,28((-1)-1)$ vs $\left.0,0((-1)-1){ }^{\circ} \mathrm{C}, \mathrm{p} 0,849\right]$, aunque resultó estadísticamente significativa para la variación de la temperatura media $[0,3$ $\left((-3)\right.$ - 4) vs $\left.-0,3((-3)-3)^{\circ} \mathrm{C}, \mathrm{p} 0,039\right]$.

Resultados similares se obtuvieron al comparar las variaciones de humedad relativa entre los días con casos de HSA y días sin los mismos, tanto en las variaciones diarias [25 (20 - 33) vs $26(19-34)$, p 0,594], como en los cambios de los valores máximos $[-1,0((-8)$ - 7) vs $1,0((-6)-7), p$ $0,244]$, mínimos $[0,0((-8)-8)$ vs $0,0((-8)-9)$, p 0,521$]$ y medios $[-1,0((-11)-9)$ vs $0,0((-10)-10), \mathrm{p} 0,381]$.

\section{Discusión}

Esta es una serie retrospectiva de ámbito hospitalario. Es decir, un porcentaje de pacientes no han sido incluidos en la misma por haber fallecido antes de llegar al hospital (que oscila entre un $8 \%$ y un 15\%, según lo publicado en algunas series ${ }^{4,8,10,11}$, aunque en la mayoría de los estudios realizados en nuestro medio se hace constar únicamente la mortalidad intrahospitalaria ${ }^{14,16}$ ). Si bien, no hay forma de saber con exactitud el porcentaje de individuos que fallecen por HSA antes de llegar al hospital, entre otras razones porque no siempre se realizan necropsias; no se refleja este aspecto adecuadamente en los informes del Instituto Anatómico Forense, o bien no tenemos acceso a los mismos. En todo caso, el objeto de este estudio es conocer la posible influencia de algunas variables meteorológicas en pacientes con HSA espontánea diagnosticada con certeza.

Son numerosos los estudios que en las últimas décadas han tratado de encontrar variables, tanto intrínsecas como extrínsecas, que se relacionen con un aumento del riesgo de 
hemorragia subaracnoidea. Entre los factores extrínsecos más frecuentemente estudiados se encuentran las variables meteorológicas.

Algunos autores abogan por una distribución estacional ${ }^{6}$, describiendo diversos patrones, sin unanimidad, con picos de incidencia en invierno ${ }^{9,22}$, en otoño ${ }^{3}$ o en primavera ${ }^{1}$, a pesar de que otros no encuentran tal influencia ${ }^{2,18,20}$. En otras series, se describen patrones de variación circadiana ${ }^{6,9,12,15,18}$ que podrían relacionarse con las variaciones en la presión arterial ${ }^{9,21}$, sin resultados concluyentes. En nuestro estudio no encontramos diferencias en la distribución estacional, salvo una tendencia al descenso en el número de casos durante la etapa estival, que no resulta estadísticamente significativa, si bien, no se analizó la distribución circadiana por carecer de datos referentes al momento de instauración del cuadro clínico.

En los estudios que analizan las diversas variables meteorológicas, los resultados son igualmente contradictorios. Disminuciones en la presión atmosférica los días previos a la hemorragia subaracnoidea se relacionan con aumentos en la incidencia de la misma ${ }^{3,13,20}$ en algunas series, mientras que otras no diferencian entre aumentos o disminuciones ${ }^{2}$ o bien, no encuentran relación ${ }^{1}$. Nuestro análisis de las variaciones de la presión atmosférica en el día de la hemorragia o respecto al día precedente, tanto en los valores medios como en los máximos y mínimos, no ha encontrado una relación significativa con el aumento de la incidencia.

Algunas series han considerado la temperatura y la humedad relativa como posibles variables concluyendo, en la mayor parte de los casos, que no suponen un factor de riesgo $0^{1,20}$ para la HSA. Nuestra serie no se relaciona con ninguna de ambas variables, aunque se observa un aumento significativo con la variación de la temperatura media respecto al día previo, cuyo significado aún queda por determinar.

A la vista de los resultados y considerando otros estudios similares ${ }^{1,20}$ la diversidad de las conclusiones podría deberse a diferencias metodológicas. Por un lado, hemos considerado las hemorragias tanto aneurismáticas como angiográficamente negativas, ya que de otra forma, era presuponer un mecanismo fisiopatológico que realmente es desconocido ${ }^{7}$. Por otro lado, el hecho de ser un análisis retrospectivo limita la interpretación de los resultados, al no disponer de datos acerca del momento de inicio de los síntomas así como la población en la que tuvieron lugar, ya que se ha asumido que todos los casos han sucedido en Alicante, aunque si bien, por la orografía de la región no existen diferencias climatológicas significativas entre las diversas poblaciones que atiende nuestro servicio, por lo que no sería estrictamente necesario especificar la localización geográfica en que se instauró el cuadro.

\section{Conclusión}

En nuestro medio las variaciones de presión atmosférica no se relacionan con un aumento de la incidencia de la hemorragia subaracnoidea, produciéndose ésta con cambios de apenas $1,7(1,3) \mathrm{hPa}$ en el día de la hemorragia. Asimismo las diferencias en los valores de temperatura y humedad, tampoco parecen influir en la distribución de los casos de hemorragia, salvo cambios de aproximadamente $0,3^{\circ} \mathrm{C}$ en la temperatura media, quedando aún por determinar el significado clínico de tal resultado. Por último, no encontramos una marcada distribución estacional, aunque se observa una paradójica tendencia al descenso en el periodo estival, a pesar del conocido aumento de población flotante que se registra en Alicante durante dicha época.

Los resultados indican la necesidad de continuar investigando posibles factores y condiciones sobre los que poder incidir y modificar el riesgo de HSA.

Nota. El ámbito de cobertura del Hospital General Universitario de Alicante, aparte de las unidades de referencia de distritos, es el establecido por la Orden de 27 de Diciembre de 1993 de la Consellería de Sanitat i Consum por la que se delimita el mapa sanitario de la Comunidad Valenciana (D.O.G.V. $\mathrm{n}^{\mathrm{o}} 2.175$, de 30-12-95) y se establece como población de referencia al Departamento 19 que incluye las poblaciones de Alicante, Agost, Moralet, Verdegás, Monforte del Cid, San Vicent del Raspeig, La Alcoraya y La Canyada.

\section{Bibliografía}

1. Beseoglu, K., Hanggi, D., Stummer, W., Steiger, H.J.: Dependence of subarachnoid hemorrhage on climate conditions: a systematic meteorological analysis from the Dusseldorf metropolitan area. Neurosurgery 2008; 62: 1033-1039.

2. Buxton, N., Liu, C., Dasic, D., Moody, P., Hope, D.T.: Relationship of aneurismal subarachnoid hemorrhage to changes in atmospheric pressure: Results of a prospective study. J. Neurosurg. 2001; 95: 391-392.

3. Chyatte, D., Chen, T.L., Bronstein, K., Brass, L.M.: Seasonal fluctuation in the incidence of intracranial aneurysm rupture and its relationship to changing climatic conditions. J. Neurosurg. 1994; 81: 525-530.

4. Echevarría, G., Gonorazky, S.E., Gáspari, M., Valussi, C., Femminini, R.: Primer episodio de hemorragia subaracnoidea no traumática. Estudio epidemiológico de su incidencia y de la hipertensión arterial como un factor de riesgo. Rev neurol. 2003; 37: 425-430.

5. Feigin, V.L., Anderson, C.S., Anderson, N.E., Broad, J.B., Pledger, M.J., Bonita, R.: Australasian Co-operative Research Group on Subarachnoid Haemorrhage Study (ACROSS) and Auckland Stroke Studies: Is there a temporal 
pattern in the occurrence of subarachnoid hemorrhage in the southern hemisphere? Pooled data from 3 large, populationbased incidence studies in Australasia, 1981 to 1997. Stroke 2001; 32: 613-619.

6. Gallerani, M., Portaluppi, F., Maida, G., et al.: Circadian and circannual rhythmicity in the occurrence of subarachnoid hemorrhage. Stroke 1996; 27: 1793-1797.

7. Greenberg, M.: Handbook of Neurosurgery. New York; Thieme, 2006; pp. 781-802.

8. Hop, J.W., Rinkel, G.J., Algra, A., Van Gijn, J.: Casefatality rates and functional outcome after subarachnoid hemorrhage a systematic review. Stroke 1997; 28: 660-664.

9. Inagawa, T., Takechi, A., Yahara, K., et al.: Primary intracerebral and aneurysmal subarachnoid hemorrhage in Izumo City, Japan. Part I: Incidence and seasonal and diurnal variations. J. Neurosurg. 2000; 93: 958-966.

10. Inagawa, T.: Trends in Incidence and Case Fatality Rates of Aneurysmal Subarachnoid Hemorrhage in Izumo City, Japan, Between 1980-1989 and 1990-1998. Stroke 2001; 32: 1499-1507.

11. Ingall, T., Asplund, K., Mähönen, M., Bonita R.: A multinational comparison of subarachnoid hemorrhage epidemiology in the WHO MONICA Stroke study. Stroke 2000; 31 : 1054-1061.

12. Kleinpeter, G., Schatzer, R., Böck, F.: Is blood pressure really a trigger for the circadian rhythm of subarachnoid hemorrhage? Stroke 1995; 26: 1805-1810.

13. Lejeune, J.P. et al.: Association of occurrence of aneurysmal bleeding with meteorologic variations in the north of France. Stroke 1994; 25: 338-341.

14. Muñoz, M.A., García, C., Muñoz, A., et al.: Proyecto EHSA: estudio de hemorragias subaracnoideas espontáneas en Andalucía. Incidencia y resultados. Rev neurol. 2003; 36: 301306.

15. Nyquist, P.A., Brown, R.D. Jr., Wiebers, D.O., Crowson, C.S., O'Fallon, W.M.: Circadian and seasonal occurrence of subarachnoid and intracerebral hemorrhage. Neurology

\section{Comentario al trabajo Influencia de los cambios de pre- sión atmosférica y otras variables meteorológicas en la incidencia de la hemorragia subaracnoidea de E. Baño Ruiz y cols.}

En este interesante trabajo Baño Ruiz y cols. demuestran cómo en su serie no existe una clara relación entre las variaciones de presión, humedad y temperatura con la aparición de casos de hemorragia subaracnoidea espontánea. Es un fenómeno bastante comentado por muchos de nosotros como, aparentemente, los casos de esta patología se agregan en el tiempo con frecuencia, sobre todo ante cambios meteorológicos. Se añade a esto la aceptación general de que la hemorragia subaracnoidea se da con
2010; 21: 14-21

2001; 56: 190-193.

16. Parkhutik, V., Lago, A., Tembl, J.I., Beltrán, A., Fuset, M.P.: Hemorragia subaracnoidea espontánea: estudio de 462 pacientes. Rev neurol. 2008; 46: 705-708.

17. Rengachary, S., Ellenbogen, R.: Principles of neurosurgery. London; Elsevier, 2005; pp. 215-219.

18. Sarah, E., Vermeer, Gabriel J. E. Rinkel, and Ale Algra: Circadian Fluctuations in Onset of Subarachnoid Hemorrhage: New Data on Aneurysmal and Perimesencephalic Hemorrhage and a Systematic Review Stroke, Apr 1997; 28: 805-808.

19. Schievink, W.I., Wijdicks, E.F., Meyer, F.B., Piepgras, D.G., Fode, N.C., Whisnant, J.P.: Seasons, snow and subarachnoid hemorrhage: Lack of association in Rochester, Minnesota. J. Neurosurg. 1995; 82: 912-913.

20. Setzer, M. et al.: The influence of barometric pressure changes and meteorological variables on the occurrence and clinical features of subarachnoid hemorrhage. Surg. Neurol. 2007; 67: 264-272.

21. Sloan, M.A., Price, T.R., Foulkes, M.A., et al.: Circadian rhythmicity of stroke onset. Intracerebral and subarachnoid hemorrhage. Stroke 1992; 23: 1420-1426.

22. Wang, Y., Levi, C.R., Attia, J.R., D’Este, C.A., Spratt, N., Fisher, J.: Seasonal variation in stroke in the Hunter Region, Australia: A 5-year hospital-based study, 1995-2000. Stroke 2003; 34: 1144-1150.

Baño-Ruiz, E.; Abarca-Olivas, J.; Duart-Clemente, M.; Ballenilla-Marco, F.; García, P.; Botella-Asunción, C.: Influencia de los cambios de presión atmosférica y otras variables meteorológicas en la incidencia de la hemorragia subaracnoidea. Neurocirugía 2010; 21: 14-21.

Correspondencia postal: Servicio de Neurocirugia. Hospital General Universitario de Alicante. C/ Pintor Baeza s/n, 03010 Alicante

E-mail: ebano@neurocirugia.com

mayor frecuencia en unas estaciones que en otras o que aparece con mayor frecuencia a unas horas del día. Sin embargo, es poca la evidencia sobre estos aparentes factores desencadenantes de la hemorragia. Por un lado, la agregación estacional no se da en este ni en otros estudios, incluyendo estudios multicéntricos nacionales ${ }^{1}$. Por otro, la aparición a unas horas del día, aunque señalada por algunas series $^{2}$, es difícil de establecer y probablemente tengan más que ver con la variación circadiana de la presión arterial, 
como pasa con otros ictus, que con cualquier variable meteorológica. Además, la evidencia sobre la relación entre la meteorología y la ruptura de los aneurismas es cambiante, dada la existencia de estudios que la apoyan y otros que sin embargo la desmienten. Quizás en este sentido cabe destacar algunos problemas metodológicos a la hora de realizar e interpretar estos estudios. Por un lado hay que asegurar que todos los casos que se detectan en el hospital son los que han sucedido durante el periodo de estudio en esa área geográfica o de referencia del hospital, dado que algunos enfermos pueden ser trasladados a hospitales de otras áreas más cercanas. Por otro lado es importante establecer el momento en el que se produce el sangrado con exactitud. Ambas consideraciones son tomadas en cuenta por los autores a la hora de discutir sus resultados.

\section{Bibliografía}

1. Lagares, A., de Toledo, P., Fernández-Alén, J.A., Ibáñez, J., Arikan, F., Sarabia, R., Ballenilla, F., Gabarros, A., Horcajadas, A., Rodríguez-Boto, G., de la Lama, A., Maillo, A., Delgado, P., Llacer, J.L., Domínguez, J., Arrese, I.: Base de datos multicéntrica de hemorragia subaracnoidea espontánea del Grupo de Trabajo de Patología Vascular de la Sociedad Española de Neurocirugía: presentación, criterios de inclusión y desarrollo de una base de datos en Internet. Neurocirugía 2008; 19: 405-415.

2. Martínez-Mañas, R., Ibáñez, G., Macho, J., Gastón, F., Ferrer, E.: Estudio de 234 pacientes con hemorragia subaracnoidea de origen aneurismático y criptogenético. Neurocirugía 2002; 13: 281-295. 